\title{
Respuesta a Factores Estimulantes de Colonias de Granulocitos en eventos de Neutropenia Febril en Oncológicos del Instituto del Cáncer Solca-Cuenca.
}

*Correspondencia:

hernandocordero@hotmail.com

Teléfono [593] 072814637

Conflicto de intereses: Los autores declaran no tener conflictos de intereses.

Fondos: Ver la página 229

Recibido: 15 Julio 2017

Aceptado: 20 Agosto 2018

Publicado: 30 Diciembre 2018

Membrete bibliográfico:

Cordero H, Staël J, Jerves $M$, Martínez F. Respuesta a Factores Estimulantes de Colonias de Granulocitos en eventos de Neutropenia Febril en pacientes Oncológicos del Instituto del Cáncer Solca-Cuenca. Rev. Oncol. Ecu 2018;28(3):219-231.

DOI: $\underline{\text { https://doi.org/10.33821/168 }}$

Copyright Cordero, et al. Este artículo es distribuido bajo los términos de Creative Commons Attribution License, el cual permite el uso y redistribución citando la fuente y al autor original.

\section{Response to Stimulating Factors of Granulocyte Colonies in Febrile Neutropenia Events in Oncological Patients of the Cancer Institute Solca-} Cuenca.

\section{Hernando Cordero ${ }^{1 *(D)}$, Juan Staël'1, Miguel Jerves², Fray Martínez¹.}

1. Facultad de Medicina, Universidad del Azuay.

2. Instituto del Cáncer SOLCA-Cuenca, Servicio de Clínica.

\section{Resumen}

Introducción: La Neutropenia Febril es una complicación potencialmente fatal del tratamiento del cáncer, relacionada con mayor morbilidad, mortalidad, disminución de dosis o retardo en los ciclos de quimioterapia, y resultados finales pobres. Estudios anteriores han demostrado el beneficio de Factor Estimulante de Colonias de Granulocitos en la reducción de tiempo de hospitalización, antibióticos intravenosos, fiebre y recuperación del conteo absoluto de neutrófilos. Se decide realizar el presente reporte ya que no existen datos respecto al manejo y respuesta al tratamiento en nuestro medio.

Métodos: El presente estudio descriptivo, retrospectivo, fue realizado en el Instituto del Cáncer SOLCA - Cuenca. Se revisaron las historias clínicas del período 2010 - 2011. Las variables analizadas fueron: número de días de hospitalización, fiebre, uso de antibióticos intravenosos, y días de recuperación de neutropenia a $>500 / \mathrm{mm}^{3}$ y $>1000 / \mathrm{mm}^{3}$.

Resultados: La estancia hospitalaria tuvo una mediana de 6 días, los días de terapia antibiótica intravenosa fueron iguales a los días de hospitalización. 79 eventos se recuperaron a un conteo absoluto de neutrófilos $>500 / \mathrm{mm}^{3}$, en una mediana de 4 días; 72 eventos se recuperaron a $>1000$ $/ \mathrm{mm}^{3}$ en una mediana de 4 días. La mayoría de los eventos se volvieron afebriles en una mediana de 1 día. 
Conclusión: Los resultados de las variables estancia hospitalaria, uso de antibióticos intravenosos y la duración de la fiebre fueron similares a los ya descritos en estudios anteriores, la recuperación del conteo absoluto de neutrófilos, fue más tardía, mostrando diferencias importantes con la bibliografía.

Palabras Claves: LEUCOPENIA, FIEBRE, FACTOR ESTIMULANTE DE COLONIAS DE GRANULOCITOS, NEUTRÓFILOS.

DOI: $10.33821 / 168$

\section{Abstract}

Introduction: Febrile Neutropenia is a potentially fatal complication of cancer treatment, related to higher morbidity, mortality, dose reduction or retard in chemotherapy cycles, and poor final outcomes. Previous studies have demonstrated the benefit of G-CSF (Granulocyte - Colony Stimulating Factors) in reduction of hospital stay, the use of intravenous antibiotics, fever and absolute neutrophil count (ANC) recovery. There is no data about the management and treatment response in our population.

Methods: This is a retrospective descriptive study, developed in SOLCA - Cuenca Cancer Institute. 83 febrile neutropenia events met the inclusion and exclusion criteria, medical records from years 2010 to 2011 were reviewed. The analyzed variables were: days of hospital stay, fever, intravenous antibiotics use, and neutropenia recovery to a level $>500 / \mathrm{mm}^{3}$ and $>1000 / \mathrm{mm}^{3}$.

Results: The median of hospital stay was 6 days, the duration of IV antibiotic therapy was the same as the days of hospital stay. 79 events recovered to an ANC $>500 / \mathrm{mm}^{3}$, with a median of 4 days; 72 events recovered to $>1000 / \mathrm{mm}^{3}$ with a median of 4 days. The majority of events became afebrile with a median of 1 day.

Conclusion: The results in the variables hospital stay, use of intravenous antibiotics and fever duration, were similar to those described in previous studies. The ANC recovery was delayed, showing important differences with cited references.

Keywords: LEUKOPENIA, FEVER, STIMULATING FACTOR OF COLONIES OF GRANULOCYTES, NEUTROPHILS.

DOI: $10.33821 / 168$

\section{Introducción}

La neutropenia febril (NF) es una complicación potencialmente fatal del tratamiento del cáncer cuando se asocia a infección y sepsis [1]. Es definida por la European Society for Medical Oncology (ESMO) como un conteo absoluto de neutrófilos (por sus siglas en Inglés ANC) $<500 / \mathrm{mm}^{3}$, o un ANC $<1000 / \mathrm{mm}^{3}$ que se predice descenderá a menos de $500 / \mathrm{mm}^{3}$ dentro de 48 horas, sumado a fiebre (temperatura oral $>38.5^{\circ} \mathrm{C}$ al menos por una hora, o dos lecturas consecutivas $>38.0^{\circ} \mathrm{C}$ por 2 horas) y signos clínicos de sepsis [2]. La U.S. National Cancer Institute ( $\mathrm{NCl}$ ) así como la Organización Mundial de la Salud (OMS-WHO) clasifican a la neutropenia según el ANC en: Grado $0\left(\geq 2000 / \mathrm{mm}^{3}\right)$, Grado $1(\geq 1500$ a $\left.<2000 / \mathrm{mm}^{3}\right)$, Grado $2\left(\geq 1000 \mathrm{a}<1500 / \mathrm{mm}^{3}\right)$, Grado $3\left(\geq 500 \mathrm{a}<1000 / \mathrm{mm}^{3}\right)$, y Grado 4 $\left(<500 / \mathrm{mm}^{3}\right)$ [3]. 
La fiebre ocurre frecuentemente durante la neutropenia inducida por quimioterapia: 10 $50 \%$ de pacientes con tumores sólidos y más del $80 \%$ de las malignidades hematológicas desarrollaran fiebre durante más de un ciclo de quimioterapia asociada con neutropenia, [4] con una infección documentada en sólo el 20 al 30 \% de ellos [5].

\section{Factores Estimulantes de Colonias de Granulocitos (G-CSF)}

Los G-CSF endógenos son glicoproteínas que actúan en las células del sistema hematopoyético estimulando su proliferación, diferenciación y activación final, son producidos por monocitos, fibroblastos y células endoteliales.

Filgrastim es un G-CSF humano producido por tecnología de ADN recombinante [6]. Es una proteína de 175 aminoácidos producida por Escherichia coli (E. coli), bacteria en la que se insertó el gen de G-CSF humano. Con un peso molecular de 18,800 daltons, la proteína tiene una secuencia de aminoácidos idéntica a la secuencia natural excepto por la adición de un $\mathrm{N}$-metionina terminal necesario para la expresión en la $\mathrm{E}$. coli ( $r$-metHuG-CSF). El producto es no glicosilado por lo que difiere de la forma aislada en las células humanas. Filgrastim ha sido evaluado en varios estudios iniciales de eficacia y seguridad, que han sido usados como referencia para la elaboración de las guías clínicas y recomendaciones de manejo actuales.

El realizado por Mayordomo et al.[7] en el años 1995, un ensayo clínico abierto, evaluó los beneficios de adicionar G-CSF y Granulocyte-Macrophage Colony Stimulating Factor (GMCSF) a la terapia antibiótica estándar en pacientes con neutropenia febril posquimioterapia; incluyeron 121, asignados a 3 grupos, uno con cada factor de crecimiento y uno placebo controlado, concluyendo que la duración de la fiebre y la neutropenia es menor, además de una tendencia a un menor costo por cuidado de cada paciente.

García-Carbonero et al [8] en un ensayo clínico multicéntrico aleatorizado en 2001, evaluó prospectivamente la eficacia de añadir G-CSF a la terapia antibiótica en pacientes con NF de alto riesgo y con tumores sólidos, abarcó 210 pacientes asignados a grupos con antibiótico y otro con antibiótico más G-CSF, demostrando que la duración de la neutropenia, la terapia antibiótica, la hospitalización y costos de hospital fueron menores.

Una revisión de Cochrane [9] en 2005, con 13 estudios y 1.518 pacientes, no mostró una influencia significativa sobre la mortalidad pero sí en la reducción de tiempo de hospitalización, y el tiempo de recuperación de neutrófilos.

\section{Manejo: G-CSF en la Neutropenia Febril}

Todo paciente requiere una evaluación inicial del riesgo de NF previo a su quimioterapia según lo sugieren las guías EORTC [1]. Se los catalogará como riesgo bajo: $<10 \%$, intermedio: $10-20 \%$ y alto: $>20 \%$, según el tipo de malignidad y el esquema de quimioterapia a utilizar, para decidir el uso de G-CSF profiláctico.

Las guías actuales para el uso de G-CSF de Estados Unidos: ASCO [10], NCCN [11], Canadá

[12], Europa: EORTC [1] y ESMO del 2006, recomiendan consistentemente un umbral de 
riesgo del $20 \%$ para la administración de rutina de G-CSF en pacientes con tumores sólidos y linfoma [13].

Los pacientes que desarrollen fiebre durante su tratamiento deben ser evaluados mediante el score MASCC [14] que valora además el riesgo de presentar bacteriemia y sepsis en pacientes con diagnóstico de neutropenia febril [15], sin embargo tiene utilidad limitada en malignidades hematológicas y pediátricas [16].

Las guías de práctica clínica para manejo de antimicrobianos en pacientes con cáncer del 2010 publicadas por el Infectious Diseases Society of America (IDSA), recomiendan también la terapia empírica urgente dentro de las primeras 2 horas de presentación de fiebre [17].

La recomendación indica usar Filgrastim $5 \mu \mathrm{g} / \mathrm{kg} /$ día de G-CSF vía subcutánea (s.c.) 24$72 \mathrm{~h}$ luego del último día de quimioterapia hasta una recuperación del ANC posnadir suficiente o estable [13].

Las guías de la $\operatorname{ESMO}[2,18]$ indican que el seguimiento diario y la frecuencia de evaluación clínica será determinada por la severidad del cuadro de NF, pero puede ser requerida hasta cada 2 a 4 horas, e incluirá patrones febriles, ANC, función medular y renal y están indicadas hasta que el paciente esté afebril o con un ANC $>500 / \mathrm{mm}^{3}$ [2].

En los pacientes con infecciones documentadas clínica o microbiológicamente, la duración de la terapia está ordenada por el microorganismo o el sitio de infección; los antibióticos apropiados se deberán continuar por lo menos durante la duración de la neutropenia $(>500$ células $/ \mathrm{mm}^{3}$ ) [17].

En los pacientes afebriles y con un ANC $>500 / \mathrm{mm}^{3}$ a las 48 horas, y catalogados de bajo riesgo sin encontrarse causa de infección, se puede considerar el cambio a antibióticos por vía oral. Si la fiebre persiste por más de 4 a 6 días se deberá iniciar tratamiento antifúngico profiláctico. En pacientes considerados de alto riesgo y sin causa identificada se reevaluará el esquema de tratamiento antimicrobiano.

A pesar de existir guías internacionales para el manejo de G-CSF y referencias sobre su efecto en el curso clínico de la patología, en nuestro país no existen datos que muestren la respuesta en nuestra población al tratamiento con estos fármacos.

El objetivo general del estudio es describir la respuesta al tratamiento con G-CSF en los eventos de NF, en el Instituto del Cáncer SOLCA - Cuenca. Se describirán como objetivos específicos: las características generales de cada evento, así como el tiempo de hospitalización, la duración de la fiebre, la terapia antibiótica intravenosa, el tiempo de recuperación del ANC, todos desde el inicio de la terapia con G-CSF. 


\section{Materiales y Métodos}

Estudio descriptivo, retrospectivo realizado en el Instituto del Cáncer SOLCA - Cuenca, a partir de las historias clínicas de los pacientes que cumplieron los criterios de inclusión, en los años 2010 y 2011.

El universo lo conforman todos los pacientes tratados con quimioterapia durante los años 2010 y 2011. La búsqueda en el sistema informático para seleccionar los eventos de NF se realizó mediante los términos: D70, correspondiente al diagnóstico de Agranulocitosis según el CIE-10, utilizado como análogo para el término NF que no consta en el listado; además se realizó la búsqueda entre los pacientes que tenían descargos de farmacia del medicamento Filgrastim. Se obtuvieron 112 pacientes, se excluyeron 52 por tratarse de eventos de Neutropenia afebril, pacientes en tratamiento profiláctico con Filgrastim, conteos de ANC mayores a 500/ $\mathrm{mm}^{3}$ al inicio, uso de Pegfilgrastim (forma comercial pegilada), y pacientes sin controles de ANC, controles de temperatura, o sin antibióticos.

La muestra definitiva se compone de 60 pacientes y 83 eventos de NF, tomando en cuenta que algunos pacientes tuvieron más de un evento los cuales se analizan por separado. Se revisaron una a una las historias clínicas electrónicas de los pacientes seleccionados desde el año 2010 al 2011, la información requerida se registró en fichas de recolección de datos. Se elaboró una base de datos inicial en el programa Microsoft Excel 2007, luego se realizó el análisis estadístico en el Software IBM SPSS Statistics Versión 19.0, utilizando medidas de tendencia central y dispersión.

\section{Definiciones}

Duración de Hospitalización: Número de días que el paciente permaneció hospitalizado en cada evento, tomando como inicio el día de administración de Filgrastim.

Duración de Fiebre: Número de días consecutivos o cualquier día subsecuente en que el paciente presentó una temperatura mayor a $37.5^{\circ} \mathrm{C}$, iniciando el día de administración de Filgrastim.

Duración de Antibióticos: Número de días de terapia antibiótica intravenosa, iniciando el día de administración de Filgrastim.

Duración de Neutropenia: Número de días que tardaron los pacientes en cada evento hasta que tuvieron un control de ANC $>500 / \mathrm{mm}^{3}$ y $>1000 / \mathrm{mm}^{3}$.

\section{Resultados}

Un total de 83 eventos de NF que cumplieron con los criterios de inclusión fueron seleccionados para el estudio. En la Tabla 1, se muestran las características generales de la población. De 83 eventos de NF, 79 se recuperaron a un conteo de neutrófilos $>500 / \mathrm{mm}^{3}$, y 72 a >1000 / $\mathrm{mm}^{3}$, más detalles en la Tabla 2. La distribución de los datos de las variables 
en el tiempo no presenta un modelo normal, por lo que los resultados se presentan en medianas, como en la bibliografía existente. El porcentaje acumulado de pacientes que se recuperaron de la neutropenia se muestra en la Figura 1, sin alcanzar una recuperación del ANC en la totalidad de eventos. El $69.9 \%$ de los eventos alcanzó una recuperación a $>2000 / \mathrm{mm}^{3}$, el $88 \%$ se recuperó a $>1000 / \mathrm{mm}^{3}$ y el $95.2 \%$ a $>500 / \mathrm{mm}^{3}$. Sólo el $4.8 \%$ no logró conteos mayores a $500 / \mathrm{mm}^{3}$ hasta el final de la evaluación.

La duración de la neutropenia fue mayor en adolescentes y adultos-jóvenes de 15 a 39 años con una media de 6.1 días, en comparación a niños de 0 a 9 años con 5.1 días. (Tabla 3). La mayoría de los eventos se volvieron afebriles en una mediana de 1 día (media 2.6 días, DS 2.8). Se aprecia entre hombres y mujeres una media de 3.3 días (DS 3.7) y 1.9 días (DS 1.4) respectivamente, ambos grupos con una mediana de 1 día. Los que más tardaron en volverse afebriles fueron los adultos de 40 a 65 años con una media de 4.3 días (DS 2.1), al contrario, quienes menos días de fiebre presentaron fueron los adolescentes de 10 a 14 años con 1.4 días en promedio (Tabla 3).

Los eventos con más días de hospitalización fueron los que tuvieron diagnóstico de Linfoma (mediana: 9 días), y quienes recibieron el esquema Hyper-CVAD (mediana: 10 días) (Tabla 4).

Tabla 1. Características Generales de 83 eventos de Neutropenia Febril en el Instituto del Cáncer SOLCA-Cuenca.

\begin{tabular}{|c|c|c|c|}
\hline \multicolumn{2}{|c|}{ Características de Pacientes } & $\mathbf{N}^{\circ}$ & $\%$ \\
\hline & & \multicolumn{2}{|c|}{$n=83)$} \\
\hline \multirow[t]{2}{*}{ Año } & 2010 & 36 & 43.4 \\
\hline & 2011 & 47 & 56.6 \\
\hline \multirow[t]{2}{*}{ Sexo } & Hombres & 41 & 49.4 \\
\hline & Mujeres & 42 & 50.6 \\
\hline \multirow[t]{5}{*}{ Edad } & 0 a 9 años & 33 & 39.8 \\
\hline & $10-14$ años & 20 & 24.1 \\
\hline & 15 a 39 años & 17 & 20.5 \\
\hline & 40 a 64 años & 10 & 12.0 \\
\hline & $>65$ años & 3 & 3.6 \\
\hline \multirow[t]{5}{*}{ Diagnósticos } & Leucemias Agudas & 56 & 67.5 \\
\hline & Linfomas & 11 & 13.3 \\
\hline & Sarcomas & 7 & 8.4 \\
\hline & Tumores Germinales & 5 & 6.0 \\
\hline & Otros* & 4 & 4.8 \\
\hline \multirow[t]{6}{*}{ Esquemas Quimioterapia } & Total XV & 45 & 54.2 \\
\hline & Antraciclina + Otros & 14 & 16.9 \\
\hline & Hyper CVAD & 11 & 13.3 \\
\hline & Sales de Platino + Otros & 7 & 8.4 \\
\hline & Sales de Platino + Antraciclinas & 5 & 6.0 \\
\hline & Otros** & 1 & 1.2 \\
\hline
\end{tabular}

* Ca. de mama, Ca. Neuroendocrino, Ca. de ovario estromal, Meduloblastoma.

** Citarabina + Etopósido 
Tabla 2. Indicadores de las variables principales en 83 eventos de Neutropenia Febril en el Instituto del Cáncer SOLCA-Cuenca

\begin{tabular}{lccccc}
\hline & Mediana & Media & DS & \multicolumn{2}{c}{ IC Media 95\% } \\
& & & & $\begin{array}{c}\text { Límite } \\
\text { inferior }\end{array}$ & $\begin{array}{c}\text { Límite } \\
\text { superior }\end{array}$ \\
\hline Hospitalización & 6.0 & 7.2 & 3.9 & 6.4 & 8.1 \\
Fiebre & 1.0 & 2.6 & 2.8 & 2.0 & 3.2 \\
Antibióticos IV & 6.0 & 7.2 & 4.0 & 6.4 & 8.1 \\
Neutropenia $<500 / \mathrm{mm} 3$ & 4.0 & 5.1 & 3.1 & 4.4 & 5.8 \\
Neutropenia $<1000 / \mathrm{mm} 3$ & 4.0 & 5.5 & 3.9 & 4.6 & 6.4 \\
\hline
\end{tabular}

Figura 1. Porcentaje acumulado de recuperación en controles de ANC en eventos de Neutropenia Febril

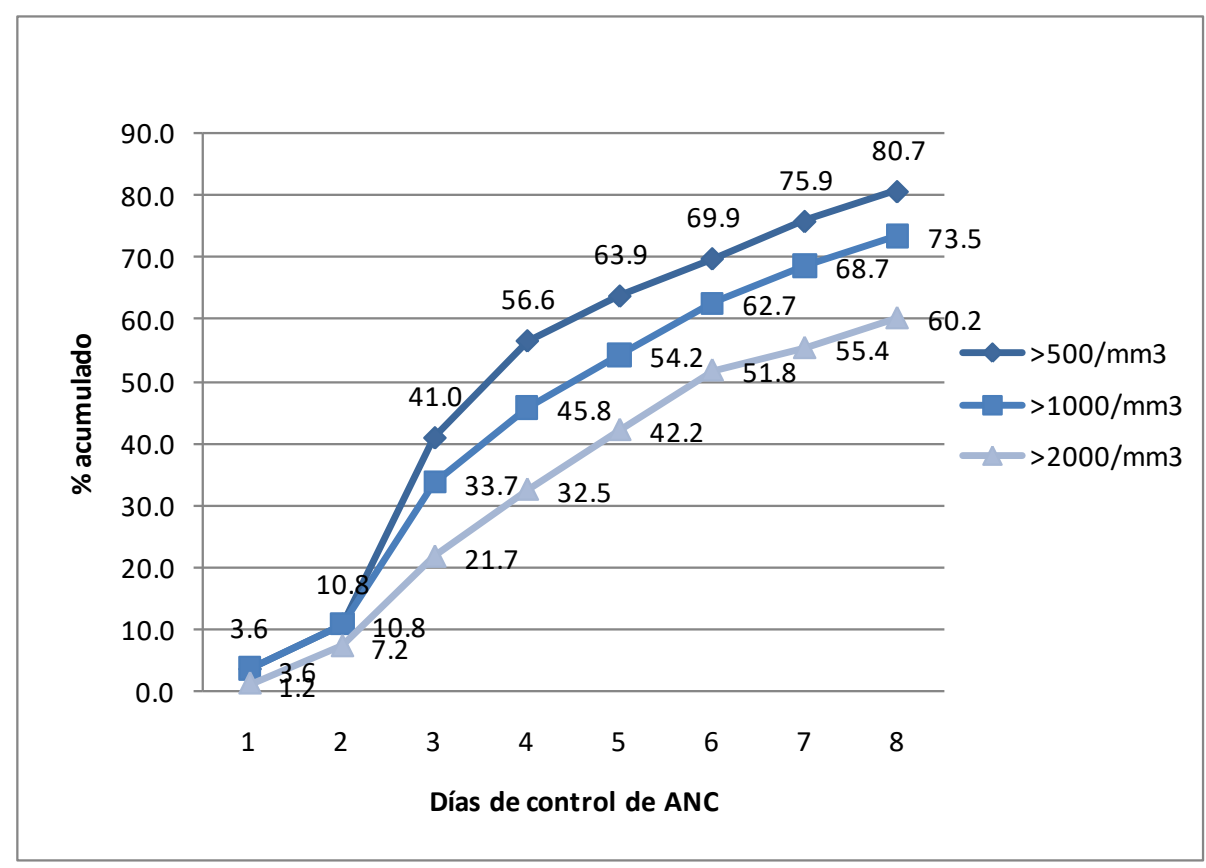


Tabla 3. Tiempo de Hospitalización, duración de fiebre, antibióticos IV y recuperación de Neutropenia en días según sexo y grupo de edad.

\begin{tabular}{|c|c|c|c|c|c|c|c|c|c|c|c|c|c|c|c|c|}
\hline & & \multicolumn{3}{|c|}{ Hos pitalización } & \multicolumn{3}{|c|}{ Fiebre } & \multicolumn{3}{|c|}{ Antibióticos IV } & \multicolumn{3}{|c|}{$\begin{array}{c}\text { Neutropenia } \\
<500 / \mathrm{mm}^{3}\end{array}$} & \multicolumn{3}{|c|}{$\begin{array}{l}\text { Neutropenia } \\
<1000 / \mathrm{mm}^{3}\end{array}$} \\
\hline & & Media & DS & Mediana & Media & DS & Mediana & Media & DS & Mediana & Media & DS & Mediana & Media & DS & Mediana \\
\hline \multirow[t]{6}{*}{ HOMBRES } & 0 a 9 años & 6.7 & 3.8 & 6.0 & 3.0 & 3.0 & 1.0 & 6.6 & 3.6 & 6.0 & 4.6 & 2.9 & 3.0 & 4.8 & 3.0 & 4.0 \\
\hline & 10 - 14 años & 5.6 & 2.4 & 5.0 & 1.6 & 1.1 & 1.0 & 5.6 & 2.4 & 5.0 & 4.9 & 2.3 & 4.0 & 5.1 & 2.4 & 4.5 \\
\hline & 15 a 39 años & 8.2 & 2.9 & 9.0 & 3.6 & 2.6 & 3.0 & 8.2 & 2.8 & 9.0 & 6.3 & 3.5 & 7.5 & 6.3 & 3.5 & 7.5 \\
\hline & 40 a 64 años & 12.0 & 7.8 & 8.0 & 8.7 & 10.0 & 5.0 & 12.0 & 7.8 & 8.0 & 7.0 & 7.9 & 4.0 & 8.3 & 8.7 & 6.0 \\
\hline & $>65$ años & 7.0 & . & 7.0 & 4.0 & . & 4.0 & 6.0 & . & 6.0 & 4.0 & . & 4.0 & 4.0 & . & 4.0 \\
\hline & TOTAL & 7.2 & 3.9 & 6.0 & 3.3 & 3.7 & 1.0 & 7.1 & 3.8 & 6.0 & 5.2 & 3.4 & 4.0 & 5.5 & 3.6 & 4.0 \\
\hline \multirow[t]{6}{*}{ MUJERES } & 0 a 9 años & 8.2 & 5.3 & 7.0 & 1.5 & 0.8 & 1.0 & 8.3 & 5.5 & 7.0 & 5.8 & 3.0 & 5.0 & 7.4 & 6.0 & 5.5 \\
\hline & 10 - 14 años & 6.3 & 3.1 & 6.0 & 1.2 & 0.4 & 1.0 & 6.3 & 3.1 & 6.0 & 4.6 & 2.3 & 4.0 & 5.2 & 2.4 & 5.0 \\
\hline & 15 a 39 años & 7.1 & 2.8 & 6.0 & 3.4 & 2.1 & 4.0 & 8.0 & 2.9 & 7.0 & 5.6 & 3.4 & 4.0 & 5.6 & 3.4 & 4.0 \\
\hline & 40 a 64 años & 6.3 & 3.0 & 6.0 & 2.4 & 1.4 & 2.0 & 5.7 & 3.8 & 6.0 & 3.5 & 2.4 & 2.5 & 3.5 & 2.4 & 2.5 \\
\hline & $>65$ años & 9.5 & 4.9 & 9.5 & 1.5 & 0.7 & 1.5 & 9.5 & 4.9 & 9.5 & 2.0 & 0.0 & 2.0 & 2.0 & 0.0 & 2.0 \\
\hline & TOTAL & 7.3 & 4.0 & 6.0 & 1.9 & 1.4 & 1.0 & 7.3 & 4.2 & 6.0 & 4.9 & 2.8 & 4.0 & 5.6 & 4.3 & 4.5 \\
\hline \multirow[t]{6}{*}{ TOTAL } & 0 a 9 años & 7.4 & 4.5 & 6.0 & 2.3 & 2.4 & 1.0 & 7.3 & 4.6 & 6.0 & 5.1 & 3.0 & 4.0 & 5.9 & 4.6 & 4.0 \\
\hline & $10-14$ años & 6.0 & 2.7 & 5.0 & 1.4 & 0.8 & 1.0 & 6.0 & 2.7 & 5.0 & 4.8 & 2.3 & 4.0 & 5.2 & 2.3 & 5.0 \\
\hline & 15 a 39 años & 7.8 & 2.8 & 7.0 & 3.5 & 2.3 & 3.0 & 8.1 & 2.8 & 9.0 & 6.1 & 3.3 & 7.0 & 6.1 & 3.3 & 7.0 \\
\hline & 40 a 64 años & 8.0 & 5.2 & 6.5 & 4.3 & 5.7 & 2.5 & 7.6 & 5.7 & 6.5 & 4.7 & 4.7 & 3.0 & 5.1 & 5.3 & 3.0 \\
\hline & $>65$ años & 8.7 & 3.8 & 7.0 & 2.3 & 1.5 & 2.0 & 8.3 & 4.0 & 6.0 & 2.7 & 1.2 & 2.0 & 2.7 & 1.2 & 2.0 \\
\hline & TOTAL & 7.2 & 3.9 & 6.0 & 2.6 & 2.8 & 1.0 & 7.2 & 4.0 & 6.0 & 5.1 & 3.1 & 4.0 & 5.5 & 3.9 & 4.0 \\
\hline
\end{tabular}

Tabla 4. Duración de Hospitalización, Fiebre, Antibióticos IV y Recuperación de Neutropenia en días según Diagnóstico y Esquema de Quimioterapia.

\begin{tabular}{|c|c|c|c|c|c|c|c|c|c|c|c|c|c|c|c|}
\hline & \multicolumn{3}{|c|}{ Hos pitalización } & \multicolumn{3}{|c|}{ Fiebre } & \multicolumn{3}{|c|}{ Antibióticos IV } & \multicolumn{3}{|c|}{$\begin{array}{c}\text { Neutropenia } \\
<500 / \mathrm{mm}^{3}\end{array}$} & \multicolumn{3}{|c|}{$\begin{array}{l}\text { Neutropenia } \\
<1000 / \mathrm{mm}^{3}\end{array}$} \\
\hline & Media & DS & Mediana & Media & DS & Mediana & Media & DS & Mediana & Media & DS & Mediana & Media & DS & Mediana \\
\hline \multicolumn{16}{|l|}{ Diagnós tico } \\
\hline Leucemias Agudas & 7.4 & 4.2 & 6.5 & 2.8 & 3.3 & 1.0 & 7.6 & 4.2 & 7.0 & 5.4 & 3.2 & 4.0 & 6.1 & 4.2 & 5.0 \\
\hline Linfomas & 8.4 & 3.5 & 9.0 & 2.2 & 1.7 & 1.0 & 7.8 & 3.9 & 9.0 & 5.1 & 3.6 & 3.5 & 5.3 & 3.7 & 4.0 \\
\hline Sarcomas & 4.6 & 1.7 & 5.0 & 1.6 & 1.0 & 1.0 & 4.4 & 1.8 & 5.0 & 4.3 & 1.5 & 4.0 & 4.3 & 1.5 & 4.0 \\
\hline Tumores Germinales & 7.0 & 3.8 & 6.0 & 1.8 & 1.3 & 1.0 & 7.0 & 3.8 & 6.0 & 4.2 & 2.9 & 3.0 & 4.2 & 2.9 & 3.0 \\
\hline Otros* & 6.3 & 2.5 & 6.5 & 3.3 & 1.7 & 3.5 & 6.0 & 2.4 & 6.0 & 2.5 & 1.3 & 2.5 & 2.5 & 1.3 & 2.5 \\
\hline \multicolumn{16}{|l|}{ Esquema de Quimioterapia } \\
\hline Total XV & 7.1 & 4.1 & 6.0 & 2.2 & 2.2 & 1.0 & 7.2 & 4.2 & 6.0 & 5.0 & 2.8 & 4.0 & 5.8 & 4.2 & 4.0 \\
\hline Antraciclinas + Otros & 7.2 & 4.6 & 6.0 & 3.3 & 5.0 & 1.0 & 6.9 & 5.0 & 6.0 & 5.1 & 4.4 & 4.0 & 5.2 & 4.8 & 4.0 \\
\hline Hyper CVAD & 8.7 & 2.4 & 10.0 & 3.3 & 2.6 & 2.0 & 8.6 & 2.3 & 9.0 & 7.0 & 2.9 & 7.0 & 7.0 & 2.9 & 7.0 \\
\hline Sales Platino + Otros & 7.3 & 4.2 & 6.0 & 2.7 & 1.6 & 2.0 & 7.1 & 4.2 & 6.0 & 3.6 & 2.5 & 3.0 & 3.6 & 2.5 & 3.0 \\
\hline Sales Platino + Antraciclinas & 5.0 & 1.6 & 5.0 & 1.8 & 1.1 & 1.0 & 4.8 & 1.8 & 5.0 & 4.4 & 1.7 & 4.0 & 4.4 & 1.7 & 4.0 \\
\hline Otros** & 8.0 & . & 8.0 & 5.0 & . & 5.0 & 8.0 & . & 8.0 & 4.0 & . & 4.0 & 6.0 & . & 6.0 \\
\hline
\end{tabular}




\section{Discusión}

Varios estudios han concluido que la adición de G-CSF a la terapia antibiótica para pacientes con NF reduce el tiempo de hospitalización, los días de uso de antibióticos intravenosos, promueven una recuperación del ANC más rápida, y acortan la duración de la fiebre. El metanálisis más reciente que incluía 13 publicaciones sobre el tema con 1.518 pacientes realizado por Clark et al.[9] en 2005 así lo establece, a pesar de que no muestra una reducción en la mortalidad general. Una tabla comparativa de resultados con otras publicaciones se presenta en la Tabla 5.

Tabla 5. Comparación de resultados con otras publicaciones.

\begin{tabular}{|c|c|c|c|c|c|c|c|}
\hline & \multirow{2}{*}{$\begin{array}{l}\text { Cordero - } \\
\text { Staël et al. }\end{array}$} & \multicolumn{2}{|c|}{$\begin{array}{c}\text { Mayordomo et al } \\
9\end{array}$} & \multicolumn{2}{|c|}{$\begin{array}{c}\text { García - } \\
\text { Carbonero et }\end{array}$} & \multicolumn{2}{|c|}{ Mitchell et al $^{21}$} \\
\hline & & G-CSF & Control & G-CSF & Control & $\mathrm{G}-\mathrm{CSF}$ & Control \\
\hline Hospitalización & 6.0 & 5.0 & 7.0 & 5.0 & 7.0 & 5.0 & 7.0 \\
\hline Fiebre & 1.0 & 1.0 & 2.0 & - & - & 2.0 & 3.0 \\
\hline Antibióticos IV & 6.0 & - & - & 5.0 & 6.0 & 5.0 & 6.0 \\
\hline ANC $<500 / \mathrm{mm}^{3}$ & 4.0 & 2.0 & 3.0 & 2.0 & 3.0 & 3.0 & 5.0 \\
\hline ANC $<1000 / \mathrm{mm}^{3}$ & 4.0 & 3.0 & 5.0 & 3.0 & 4.0 & - & - \\
\hline
\end{tabular}

La mediana de la duración de hospitalización luego de la administración de Filgrastim en nuestro estudio fue 6 días, mayor a los resultados reportados por Mayordomo et al. [7], quien mostró una mediana de 5 días tanto en los grupos de G-CSF y GM-CSF, comparado a la mediana de 7 días en el grupo de placebo. Estos resultados son similares a los publicados por García-Carbonero et al [8]; pero que no incluyó pacientes con leucemias ni quimioterapia mieloablativa.

En otro estudio doble ciego placebo controlado en población pediátrica menor de 17 años realizado por Mitchell et al. [19], que incluyó 186 eventos de NF, se reportó una mediana de hospitalización de 5 días vs. 7 en el grupo placebo. En este reporte se presentó una mediana de 6 días para el grupo de 0 a 9 años, y de 5 días para el grupo de 10 a 14 años, ambas similares o algo mayores a las del estudio mencionado, pero mejores que las del grupo placebo.

Estos eventos mostraron una mediana de duración de fiebre de 1 día, y una media de 2.6 días, Mayordomo et al. [7] reporta un resultado similar, frente a 2 días en el grupo placebo. El tiempo de duración de fiebre y el tiempo que tomó dejar los antibióticos intravenosos fueron pobremente reportados en los estudios revisados por Clark et al. [9], salvo GarcíaCarbonero et al. [8] que reporta la terapia con antibióticos IV con una mediana de 5 días frente a 6 días en el grupo control, siendo estadísticamente significativa.

Es necesario mencionar que la fidelidad de la evaluación de la fiebre puede haberse visto afectada por la administración de medicación antipirética a ciertos pacientes. 
Los antibióticos intravenosos fueron administrados durante toda la hospitalización en todos los eventos con una mediana de 6 días, a pesar de que en las guías de manejo de neutropenia febril se establece que se puede cambiar la vía de administración cuando el ANC supere los $500 / \mathrm{mm}^{3}$, lo que en nuestro estudio sucedió en una mediana de 4 días [17].

La duración de la neutropenia grado $4\left(<500 / \mathrm{mm}^{3}\right)$ y grado $3\left(<1000 / \mathrm{mm}^{3}\right)$ tienen una mediana de 4 días. En Mayordomo et al. [7], la mediana de neutropenia grado 4 fue 2 días, con 3 días en grupo placebo, y para neutropenia de grado 3 la mediana fue 3 días en el grupo de tratamiento frente a 5 días en el grupo placebo, siendo una diferencia estadísticamente significativa $(P<0.001)$, similares a los resultados de García-Carbonero et al [8]. Presumiblemente la diferencia encontrada en nuestro estudio se debe a que los controles del ANC no fueron tomados con la frecuencia diaria como en los dos estudios mencionados, sino según el juicio clínico, debido a que no está protocolizado el intervalo de controles lo que puede ocultar una mejoría más temprana.

En este estudio se recogieron datos relacionados al diagnóstico y al esquema de quimioterapia administrado, sin embargo en la mayoría de estudios sobre el tema no se hace una diferenciación ni comparación de estos datos con las variables en los resultados finales Tabla 4.

El diseño observacional retrospectivo de nuestro estudio conlleva limitaciones importantes al no tener establecido un protocolo con intervalo de controles del ANC y el registro de la evolución de la fiebre; como se lo ha realizado en estudios con diseños experimentales. Lo que al momento del análisis estadístico puede ser la causa de los resultados no concordantes con la bibliografía internacional, sin embargo, los resultados que se aportan motivan a la aplicación de protocolos específicos que posibiliten la realización de estudios de mayor alcance.

\section{Conclusiones}

El presente estudio pretendió mostrar la respuesta a G-CSF en eventos de NF en nuestra población, y comparar con los estudios iniciales en los que se han fundamentado las recomendaciones y guías clínicas actuales. Los resultados referentes a estancia hospitalaria, uso de antibióticos intravenosos y la duración de la fiebre fueron similares a los ya descritos en estudios anteriores. Sin embargo, la principal medida de respuesta, recuperación del ANC, fue más tardía, mostrando diferencias importantes con la bibliografía. Creemos que eso se debe a la falta de un protocolo de control que pudiera haber mostrado datos más fidedignos.

\section{Agradecimientos}

Se reconoce a las personas que participaron indirectamente en el estudio tales como el personal técnico y otras en general del Instituto Oncológico Solca-Cuenca. 
Nota del Editor

La Revista Oncología Ecu permanece neutral con respecto a los reclamos jurisdiccionales en mapas publicados y afiliaciones institucionales.

\section{Información adicional}

\section{Abreviaturas}

ANC: Recuento absoluto de neutrófilos.

ASCO: American Society of Clinical Oncology.

ESMO: European Society for Medical Oncology.

G-CSF: Factores Estimulantes de Colonias de Granulocitos.

$\mathrm{NCl}$ : National Cancer Institute.

NF: netropenia febril.

NCCN: National Comprehensive Cancer Network.

Archivos Adicionales

Ninguno declarado por los autores.

Fondos

Los fondos de la investigación fueron propios de los autores del presente artículo. Los autores principales realizaron este estudio como Trabajo de Graduación previo a la obtención de Título de Médico en la Facultad de Medicina de la Universidad del Azuay y no tienen relación con ninguna casa farmacéutica que produzca y/o comercialice el fármaco en cuestión.

\section{Disponibilidad de datos y materiales}

Existe la disponibilidad de datos bajo solicitud al autor de correspondencia. No se reportan otros materiales.

\section{Contribuciones de los autores}

HC, JS, realizaron la idea de investigación, revisión bibliográfica, recolección de datos, escritura del artículo. MJ realizó el análisis crítico del artículo. FM realizó la dirección del estudio y el análisis estadístico. Todos los autores leyeron y aprobaron la version final del manuscrito.

Aprobación de ética y consentimiento para participar 
No aplica ya que es un estudio retrospectivo

Consentimiento para publicación

No aplica.

Información de los autores

Hernando Cordero, Médico General, Universidad del Azuay. Correo electrónico: hernandocordero@hotmail.com. (iD) https://orcid.org/0000-0002-8300-7704

Juan Staël, Médico General, Universidad del Azuay. Correo electrónico: juanstael88@gmail.com

Miguel Jerves, Médico Tratante y jefe del servicio de clínica del Instituto del Cáncer SOLCACuenca. Correo electrónico: mjerves@uazuay.edu.ec

Fray Martínez, Asesor Estadístico Facultad de Medicina Universidad del Azuay, Instituto del Cáncer SOLCA-Cuenca. Correo electrónico: fraymartinezreyes@hotmail.com

Revisiones por pares

Acceda a la revisión de pares académicos en el siguiente enlace: https://publons.com/review/3729652/

\section{Referencias}

Abreviaturas en la referencias DOI: Digital Object Identifier

PMID: PubMed Identifier

SU: Short URL
1. Aapro MS, Bohlius J, Cameron D. 2010 update of EORTC guidelines for the use of granulocyte-colony stimulating factor to reduce the incidence of chemotherapy-induced febrile neutropenia in adult patients with lymphoproliferative disorders and solid tumours. European journal of cancer (Oxford, England: 1990). 2011;47(1):8-32. PMID: 21095116.

2. de Naurois J, Novitzky-Basso I, Gill MJ. Management of febrile neutropenia: ESMO Clinical Practice Guidelines. Annals of oncology : official journal of the European Society for Medical Oncology / ESMO. 2010;21(S5):v252-6. PMID: 20555092.

3. Gascón P, Aapro M, Ludwig H. Background and methodology of MONITOR-GCSF, a pharmacoepidemiological study of the multi-level determinants, predictors, and clinical outcomes of febrile neutropenia prophylaxis with biosimilar granulocyte-colony stimulating factor filgrastim. Critical reviews in oncology/hematology. 2011;77(3):184-97. PMID: 20189821.

4. Klastersky J. Management of fever in neutropenic patients with different risks of complications. Clinical infectious diseases : an official publication of the Infectious Diseases Society of America. 2004;39 Suppl 1(Suppl 1):S32-7. PMID: 15250018.

5. Ramphal R. Changes in the etiology of bacteremia in febrile neutropenic patients and the susceptibilities of the currently isolated pathogens. Clinical infectious diseases : an official publication of the Infectious Diseases Society of America. 2004;39(S1):S25-31. PMID: 15250017. 
6. Buxeraud J, Skrzypek A. Neupogen ${ }^{\circledR}$ - filgrastim. Actualités Pharmaceutiques. 2008;47(470):53-56 Disponible en: http://linkinghub.elsevier.com/retrieve/pii/S0515370008703124.

7. Mayordomo JI, Rivera F, Díaz-Puente MT. Improving treatment of chemotherapy-induced neutropenic fever by administration of colony-stimulating factors. Journal of the National Cancer Institute. 1995;87(11):803-8. PMID: 7540696.

8. García-Carbonero R, Mayordomo JI, Tornamira MV. Granulocyte colony-stimulating factor in the treatment of high-risk febrile neutropenia: a multicenter randomized trial. Journal of the National Cancer Institute. 2001;93(1):31-8. PMID: 11136839.

9. Clark O a C, Lyman GH, Castro A, Clark LGO, Djulbegovic B. Colony-stimulating factors for chemotherapy-induced febrile neutropenia: a meta-analysis of randomized controlled trials. Journal of clinical oncology : official journal of the American Society of Clinical Oncology. 2005;23(18):4198-214. PMID: 15961767

10. Smith TJ, Khatcheressian J, Lyman GH. 2006 Update of Recommendations for the Use of White Blood Cell Growth Factors: an Evidence-Based Clinical Practice Guideline. Journal of clinical oncology : official journal of the American Society of Clinical Oncology. 2006;24(19):3187-205. PMID: 16682719.

11. Crawford J, Allen J, Armitage J. Myeloid growth factors. Journal of the National Comprehensive Cancer Network : JNCCN. 2011;9(8):914-32. PMID: 21900221.

12. Kouroukis CT, Chia S, Verma S. Canadian supportive care recommendations for the management of neutropenia in patients with cancer. Current oncology (Toronto, Ont.). 2008;15(1):9-23. PMID: 2259432.

13. Crawford J, Caserta C, Roila F. Hematopoietic growth factors: ESMO Clinical Practice Guidelines for the applications. Annals of oncology : official journal of the European Society for Medical Oncology / ESMO. 2010;21 Suppl 5(Supplement 5):v248-51. PMID: 20555091.

14. Klastersky J, Paesmans M, Rubenstein EB, et al. The Multinational Association for Supportive Care in Cancer risk index: A multinational scoring system for identifying low-risk febrile neutropenic cancer patients. Journal of clinical oncology: official journal of the American Society of Clinical Oncology. 2000;18(16):3038-51. PMID: 10944139.

15. Kuderer NM, Dale DC, Crawford J, Lyman GH. Impact of primary prophylaxis with granulocyte colonystimulating factor on febrile neutropenia and mortality in adult cancer patients receiving chemotherapy: a systematic review. Journal of clinical oncology : official journal of the American Society of Clinical Oncology. 2007;25(21):3158-67. PMID: 17634496.

16. Klastersky J, Awada A, Paesmans M, Aoun M. Febrile neutropenia: a critical review of the initial management. Critical reviews in oncology/hematology. 2011;78(3):185-94. PMID: 20413323

17. Freifeld AG, Bow EJ, Sepkowitz K. Clinical practice guideline for the use of antimicrobial agents in neutropenic patients with cancer: 2010 update by the infectious diseases society of america. Clinical infectious diseases: an official publication of the Infectious Diseases Society of America. 2011;52(4):e56-93. PMID: 21258094.

18. Marti FM, Cullen MH, Roila F. Management of febrile neutropenia: ESMO clinical recommendations. Annals of oncology: official journal of the European Society for Medical Oncology / ESMO. 2009;20 Suppl 4(Supplement 4):166-9. PMID: 19454445.

19. Mitchell PL, Morland B, Stevens MC. Granulocyte colony-stimulating factor in established febrile neutropenia: a randomized study of pediatric patients. Journal of clinical oncology : official journal of the American Society of Clinical Oncology. 1997;15(3):1163-70. PMID: 9060560. 